\title{
Correction to: Geographic distribution of live births and infant mortality from congenital anomalies in Brazil, 2012-2017
}

\author{
Luzivan Costa Reis ${ }^{1}$. Wesley Luciano Kaizer ${ }^{2}$ Juliano André Boquett ${ }^{1,3}$ \\ Published online: 3 March 2021 \\ (C) Springer-Verlag GmbH Germany, part of Springer Nature 2021
}

\section{Correction to: Journal of Community Genetics https://doi.org/10.1007/s12687-021-00509-4}

The published online version contains several mistakes as given below.

1. In the abstract, the final two sentences are tripled (lines 1018). The sentences are:

"The description and analysis of data such as those carried out in this work are relevant to health systems and can be of great use in the formulation of public health campaigns and policies, in addition to informing and educating professionals and the population. The management of clinical actions must consider all social, economic, geographic and epidemiological factors."

2. Many sentences are in poor quality English in all sections after this accidental translation of my browser (Introduction,
Materials and Methods, Results and Discussion). The acronym CA often appears as AC.

3. The accidental translation caused almost all references to be incorrect.

4. The Brazilian governmental databases that we used in this study (such as SINASC and DATASUS) are translated incorrectly.

5. The authors' affiliation is also inaccurately presented in English.

The original article has been corrected.

The online version of the original article can be found at https://doi.org/ 10.1007/s12687-021-00509-4

Luzivan Costa Reis

luzivanreis@gmail.com

1 Post-graduate Program in Genetics and Molecular Biology,

Department of Genetics, Universidade Federal do Rio Grande do Sul, Porto Alegre, RS 91501-970, Brazil

2 Post-graduate Program in Informatics, Department of Informatics, Universidade Federal do Rio Grande do Sul, Porto Alegre, RS 91501-970, Brazil

3 Post-graduate Program in Child and Adolescent Health, Faculty of Medicine, Universidade Federal do Rio Grande do Sul, Porto Alegre, RS 90035-003, Brazil 